\title{
Auxetic behaviour from connected different-sized squares and rectangles
}

\author{
By Joseph N. Grima*, Elaine Manicaro and Daphne Attard \\ Faculty of Science, University of Malta, Msida MSD 2080, Malta
}

\begin{abstract}
Auxetic materials exhibit the unusual property of becoming fatter when uniaxially stretched and thinner when uniaxially compressed (i.e. they exhibit a negative Poisson ratio; NPR), a property that may result in various enhanced properties. The NPR is the result of the manner in which particular geometric features in the micro- or nanostructure of the materials deform when they are subjected to uniaxial loads. Here, we propose and discuss a new model made from different-sized rigid rectangles, which rotate relative to each other. This new model has the advantage over existing models that it can be used to describe the properties of very different systems ranging from silicates and zeolites to liquid-crystalline polymers. We show that such systems can exhibit scale-independent auxetic behaviour for stretching in particular directions, with Poisson's ratios being dependent on the shape and relative size of different rectangles in the model and the angle between them.
\end{abstract}

Keywords: auxetic; negative Poisson's ratio; zeolites; silicates; polymers

\section{Introduction}

Unless a material has a zero Poisson ratio, it will change its thickness when it is uniaxially stretched or compressed. In fact, conventional materials having a positive Poisson ratio are observed to get thinner when stretched and fatter when compressed in a uniaxial direction. Although such behaviour is assumed to be the norm, the classical theory of elasticity suggests that Poisson's ratio need not be positive. In fact, Poisson's ratio for isotropic three-dimensional materials ranges from -1 to 0.5 .

In recent years, there were significant developments in materials that exhibit a negative Poisson ratio (NPR), commonly known as auxetic (Evans et al. 1991). Such developments include work on cellular systems (Gibson et al. 1982; Gibson \& Ashby 1997), foams (Lakes 1987; Evans et al. 1994; Scarpa et al. 2004a; Bezazi \& Scarpa 2006; Grima et al. 2009a), polymers (Caddock \& Evans 1989; Evans et al. 1991, 1995; Baughman \& Galvao 1993; Grima \& Evans 2000a,b; Alderson et al. 2001; He et al. 2005a,b; Ravirala et al. 2005), metals (Baughman et al. 1998), silicates (Keskar \& Chelikowsky 1992; Yeganeh-Haeri et al. 1992;

*Author for correspondence (joseph.grima@um.edu.mt).

Electronic supplementary material is available at http://dx.doi.org/10.1098/rspa.2010.0171 or via http://rspa.royalsocietypublishing.org. 
Kimizuka et al. 2000; Alderson \& Evans 2001, 2002; Alderson et al. 2004, 2005) and zeolites (Grima 2000; Grima et al. 2000, 2005b, 2007c, 2009b; Sanchez-Valle et al. 2005; Williams et al. 2007).

Auxeticity can bring about various enhanced properties that include highenergy absorption properties (Bezazi \& Scarpa 2006; Alderson \& Alderson 2007), increased indentation resistance (Lakes \& Elms 1993; Alderson 1999; Evans \& Alderson 2000), enhanced sound and vibration absorption properties (Scarpa \& Tomlinson 2000; Scarpa \& Smith 2004; Scarpa et al. 2004a,b, 2005) and the ability to form dome-shaped structures (Lakes 1987; Evans et al. 1991; Alderson 1999; Evans \& Alderson 2000) and to act as smart filters (Alderson et al. 1998a, 2001; Grima et al. 2000; Ravirala et al. 2005).

Research suggests that auxeticity can be explained in terms of geometric models, which describe how particular geometric features in the micro- or nanostructure of the materials deform when they are subjected to uniaxial loads. Auxeticity is also known to be a scale-independent property, which means that the same 'geometry/deformation mechanism' may be found to operate in systems ranging from macroscale to nano (atomic) scale. As a result of this, research in the field of auxetics often focuses on the analysis of mechanistic models, which result in auxetic behaviour, and in recent years, various geometry-based models that lead to auxeticity by deforming through particular deformation mechanisms have been proposed. These include models based on conventional honeycombs deforming through stretching (Evans et al. 1995; Masters \& Evans 1996), re-entrant honeycombs deforming through hinging and/or flexure (Gibson et al. 1982; Evans et al. 1995; Masters \& Evans 1996; Gibson \& Ashby 1997), chiral honeycombs (Prall \& Lakes 1997; Grima 2000; Spadoni et al. 2005; Grima et al. 2008a,b) and two-/three-dimensional rotating rigid/semi-rigid units (Grima et al. 1999, 2004, 2005a,b, 2006, 2007a, 2008a,b; Grima 2000; Grima \& Evans $2000 a$; Ishibashi \& Iwata 2000; Alderson \& Evans 2001, 2002; Alderson et al. 2004, 2005; Attard \& Grima 2008; Attard et al. 2009a,b). In addition, at smaller length scales, in particular, the micro-, meso- and nanoscale, other effects such as defects and disorder may become important and may affect auxeticity (Gaspar et al. 2003; Gaspar 2008; Horrigan et al. 2009), although in several cases, ordered macroscale models have been found to be very useful to account for observed auxeticity qualitatively and/or quantitatively.

Rotating polygons were first studied by Grima et al. (1999), who studied rotating square structures and used them to explain the NPR in various zeolites. This area of research was further developed through the study of auxeticity of rotating congruent rectangles (Grima et al. 2004, 2005a,b), equilateral triangles (Grima \& Evans 2006), rhombi (Attard \& Grima 2008; Grima et al. 2008a,b) and parallelograms (Williams et al. 2007; Grima et al. 2008a,b; Attard et al. 2009a). In the case of rotating squares, Poisson's ratio was shown to be -1 , irrespective of the direction of loading and dimensions of the squares. This work was of particular significance in view of the many naturally occurring crystalline materials, which have geometric features similar to the 'rotating squares' model, including the zeolite natrolite, which was recently confirmed to be auxetic (Sanchez-Valle et al. 2005; Grima et al. 2007c, 2009b; Williams et al. 2007). It was also shown that rectangles of the same shape and size can be connected through hinges at their vertices in two different ways: type I, in which the rectangles are connected in such a way that the empty spaces form rhombi, or type II, in which the empty 


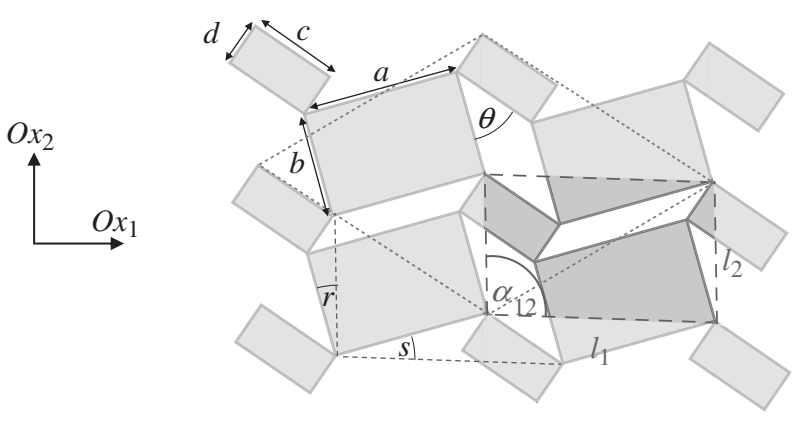

Figure 1. The system made from connected different-sized rectangles of dimensions $a \times b$ and $c \times d$ (denoted by $[a \times b, c \times d]$ ) discussed in this paper. Note that this system may be described in terms of two unit cells (UC1 and UC2) and in the derivation presented here, the system is aligned in the $O x_{1}-O x_{2}$ plane in such a way that the side of the unit cell UC2 of length $l_{2}$ is always parallel to the $\mathrm{Ox}_{2}$ direction. Grey dashed lines, UC1 and long dashed lines, UC2.

spaces are parallelograms. Grima et al. $(2004,2005 a, b)$ have shown that the type II rectangles are two-dimensionally isotropic with a Poisson ratio of -1 , whereas the type I rectangles are anisotropic with a Poisson ratio being also dependent on the shape of the rectangles and the hinging angle between adjacent rectangles. Once again, such patterns and deformation mechanisms can be found in naturally occurring auxetics, where, for example, the type II rotating 'rectangles model' has been shown to be manifested in the naturally occurring silicate $\alpha$-cristobalite.

Although this work had marked an important step forward as it highlighted the role of shape, size and connectivity of rectangles on the mechanical properties, in particular, Poisson's ratios, it was limited due to the fact that all of the rectangles were required to be of equal size and thus could only be applied to very particular and highly idealized systems. In particular, one may consider a more general system that is made from two types of rectangles of dimensions $a \times b$ and $c \times d$, which may be denoted by $[a \times b, c \times d]$, as illustrated in figure 1, of which Grima's type I and type II 'rotating rectangles' models are particular cases. (The type I systems may be achieved by letting $c=b$ and $d=a$, i.e. structure $[a \times b, b \times a]$, whereas the type II system is achieved by letting $c=a$ and $d=b$, i.e. structure $[a \times b, a \times b]$.)

In view of all this, here we present a model that can predict the behaviour of a system made from rigid rectangles of dimensions $a \times b$ and $c \times d$ connected together at their vertices through simple hinges, as illustrated in figures 1 and 2 with the aim of predicting the extent of auxeticity of such systems. Special cases arising from such systems are also discussed.

\section{Analytical model}

A space-filling tessellation is formed by connecting each $a \times b$ rectangle to four $c \times d$ rectangles, as shown in figure 1. As illustrated in figure 2 (see animation in the electronic supplementary material), stretching of such systems in particular directions will result in a relative rotation of the rectangles. This may result in 


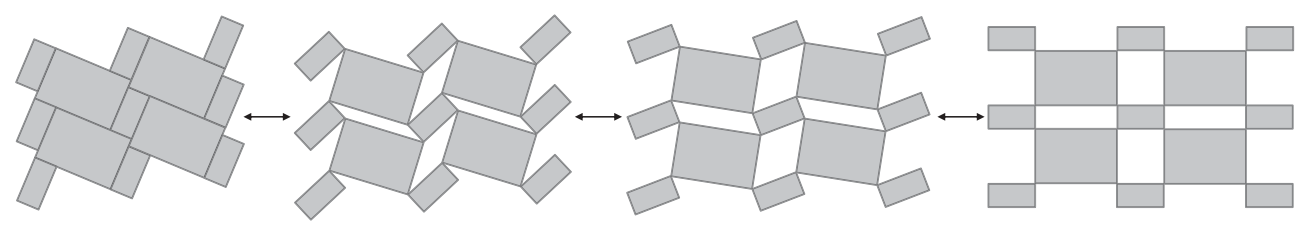

Figure 2. Illustration of the type of deformations that may be obtained from rigid rectangles connected together at their vertices through flexible hinges as a result of uniaxial loading in the $O x_{1}$ direction. Note that as the structure is stretched in the $O x_{1}$ direction, the rectangles rotate relative to each other and extend in the $\mathrm{Ox}_{2}$ direction, hence the NPR. This is also accompanied by a shearing of the structure. An animation of this figure is also provided (Anim-2.gif, electronic supplementary material).

a more open structure (hence the NPR), if one assumes that the different-sized rectangles are perfectly rigid, but are connected to each other through simple hinges, which only permit relative rotation of the rectangles. Here, it should be noted that although this paper derives Poisson's ratio in the setting of a uniaxial stretching/compression test, Poisson's ratio, like any other elastic constant, will have an effect on any stress/strain state of the system.

Note that although the rectangles can be connected in two different ways such that the resulting empty spaces between the rectangles are either parallelograms of dimensions $a \times c$ and $b \times d$ or of $a \times d$ and $b \times c$, these two cases are obviously identical as the length of the sides is chosen arbitrarily, with the result that one need not speak about type I rectangles or type II rectangles in such cases.

This tessellation illustrated in figure 1 can be described by either of the two unit cells, which have a different orientation in the global $\mathrm{Ox}_{1}-\mathrm{Ox}_{2}$ coordinate system and are being highlighted as 'UC1' and 'UC2'. Note that the UC2 unit cell is the smallest unit cell and contains only two rectangles, one of each type. In contrast, the unit cell UC1 contains four rectangles, i.e. two of each type of rectangles. Also note that these two unit cells are related such that the diagonal of the unit cell UC2 coincides with one of the sides of the unit cell UC1. In this study, the mechanical properties will be described using the unit cell UC2.

In our derivation, it shall be assumed that the structure is aligned in the $O x_{1}-O x_{2}$ space in such a way that the unit cell side of length $l_{2}$ is always parallel to the $O x_{2}$ direction, whereas the other unit cell side of length $l_{1}$ can assume any direction. Under such assumptions, the projections of the parallelogramic unit cell $\mathrm{UC} 2$ in this orientation along the $O x_{i}$ directions are given by

$$
X_{11}=l_{1} \sin \alpha_{12}
$$

and

$$
X_{22}=l_{2},
$$

where $X_{11}$ is the projection of the unit cell in the $O x_{1}$ direction, $X_{22}$ is the projection of the unit cell in the $O x_{2}$ direction and $\alpha_{12}$ is the internal angle of the unit cell $\left(\alpha_{12}=90-r+s\right.$, where $r$ and $s$ are the angles shown in figure 1$)$. The lengths $l_{1}$ and $l_{2}$ and the angle $\alpha_{12}$ may be expressed in terms of geometric parameters $a, b, c$ and $d$ (the side lengths of the rectangles, which are assumed to be constants in this derivation) and the angle $\theta$ (the angle between the rectangles, 
which is a variable), and are given by

and

$$
\begin{aligned}
l_{1} & =\sqrt{a^{2}+c^{2}+2 a c \sin (\theta)}, \\
l_{2} & =\sqrt{b^{2}+d^{2}+2 b d \sin (\theta)} \\
\sin \alpha_{12} & =\frac{1}{l_{1} l_{2}}[a b+c d+\sin (\theta)(a d+c b)] .
\end{aligned}
$$

Note that in the general case, the internal angle of the unit cell $\alpha_{12}$ is dependent on $\theta$. This implies that, in general, the unit cell shears upon deformation, i.e. a change in $\theta$ as a result of an applied stress in the $O x_{1}$ or $O x_{2}$ direction will result in strains in the $O x_{1}$ and $O x_{2}$ directions as well as a shear strain. In two dimensions, the mechanical properties of such system can be described by a $3 \times 3$ compliance matrix $\mathbf{S}$, which relates the applied stress $\boldsymbol{\sigma}$ to the resultant strain $\varepsilon$ according to the following relationship:

$$
\varepsilon=\mathbf{S} \boldsymbol{\sigma}
$$

where $\mathbf{S}$ is of the form (Daniel \& Ishai 1994)

$$
\mathbf{S}=\left(\begin{array}{ccc}
\frac{1}{E_{1}} & \frac{-\nu_{21}}{E_{2}} & \frac{\eta_{31}}{G_{12}} \\
\frac{-\nu_{12}}{E_{1}} & \frac{1}{E_{2}} & \frac{\eta_{32}}{G_{12}} \\
\frac{\eta_{13}}{E_{1}} & \frac{\eta_{23}}{E_{2}} & \frac{1}{G_{12}}
\end{array}\right),
$$

where $\nu_{12}$ and $\nu_{21}$ are Poisson's ratios for loading in the $O x_{1}$ and $O x_{2}$ directions, respectively, $E_{1}$ and $E_{2}$ are Young's moduli in the $O x_{1}$ and $O x_{2}$ directions, respectively, and $G_{12}$ is the shear modulus in the $O x_{1}-O x_{2}$ plane. $\eta_{13}, \eta_{31}, \eta_{23}$ and $\eta_{32}$ are the shear coupling coefficients defined as

$$
\eta_{i 3}=\frac{\mathrm{d} \gamma}{\mathrm{d} \varepsilon_{i}} \quad \text { and } \quad \eta_{3 i}=\frac{\mathrm{d} \varepsilon_{i}}{\mathrm{~d} \gamma}, \quad i=1,2,
$$

where $\mathrm{d} \gamma$ is the shear strain and $\mathrm{d} \varepsilon_{i}$ are the normal strains in the $O x_{i}$ directions $(i=1,2)$.

In general, the unit cell may shear upon application of only normal strains in the $O x_{i}$ directions $(i=1,2)$, a property that results in non-zero shear coupling coefficients (cases in which the internal angle $\alpha_{12}$ of the unit cell is dependent on $\theta)$, such that, in general, one needs to derive six independent elastic constants ( $\mathbf{S}$ is a symmetric matrix), e.g. $E_{i}, \nu_{i j}, G_{12}$ and $\eta_{i j}$, to fully characterize a system. However, this is not always the case, and there are certain configurations for which the internal angle of the unit cell is independent of $\theta$, in which cases, the coupling coefficients have a null value. For example, there is no shearing of the unit cell in the type II rectangles $[a \times b, a \times b]$, a system that has isotropic $\nu_{12}=-1$ (Grima et al. 2004, 2005a,b). 
(a) Strains in the $O x_{\mathrm{i}}$ directions and on-axis Poisson ratios

Poisson's ratio for loading in the $O x_{i}$ direction is given by

$$
\nu_{i j}=-\frac{\mathrm{d} \varepsilon_{j}}{\mathrm{~d} \varepsilon_{i}}, \quad i, j=1,2,
$$

where $\mathrm{d} \varepsilon_{i}$ is an infinitesimally small strain in the $O x_{i}$ direction given by the ratio of the infinitesimally small change in the unit cell dimension $\mathrm{d} X_{i i}$ to the unit cell dimension $X_{i i}$

$$
\mathrm{d} \varepsilon_{i}=\frac{\mathrm{d} X_{i i}}{X_{i i}}, \quad i=1,2 .
$$

Assuming that the only deformation mechanism is hinging, the geometry of the systems is dependent on the single variable $\theta$ and hence the strains can be re-written as

$$
\mathrm{d} \varepsilon_{i}=\frac{1}{X_{i i}} \frac{\mathrm{d} X_{i i}}{\mathrm{~d} \theta} \mathrm{d} \theta, \quad i=1,2 .
$$

By substituting for $l_{1}, l_{2}$ and $\sin \left(\alpha_{12}\right)$ in equations (2.1) and (2.2) and differentiating with respect to $\theta$, the strains along the $O x_{1}$ and $O x_{2}$ directions are found to be

$$
\mathrm{d} \varepsilon_{1}=\frac{\cos (\theta)\left[a d^{3}+c b^{3}+b d \sin (\theta)(a d+c b)\right] \mathrm{d} \theta}{\left[b^{2}+d^{2}+2 b d \sin (\theta)\right][a b+c d+\sin (\theta)(a d+c b)]}
$$

and

$$
\mathrm{d} \varepsilon_{2}=\frac{b d \cos (\theta) \mathrm{d} \theta}{b^{2}+d^{2}+2 b d \sin (\theta)} .
$$

Hence, Poisson's ratio $\nu_{12}$ for loading in the $O x_{1}$ direction and Poisson's ratio $\nu_{21}$ for loading in the $\mathrm{Ox}_{2}$ direction can be written as

$$
\begin{aligned}
\nu_{12} & =\left(\nu_{21}\right)^{-1}=-\frac{\mathrm{d} \varepsilon_{2}}{\mathrm{~d} \varepsilon_{1}}=\frac{-b d[a b+c d+\sin (\theta)(a d+c b)] \cos (\theta)}{\left[a d^{3}+c b^{3}+b d \sin (\theta)(a d+c b)\right] \cos (\theta)} \\
& = \begin{cases}\frac{-b d[a b+c d+\sin (\theta)(a d+c b)]}{\left[a d^{3}+c b^{3}+b d \sin (\theta)(a d+c b)\right]}, & \text { for } 0^{\circ} \leq \theta \leq 180^{\circ}, \quad \theta \neq 90^{\circ}, \\
L, & \text { for } \theta=90^{\circ},\end{cases}
\end{aligned}
$$

where $L$ is defined as

$$
L=\lim _{\theta \rightarrow 90^{-}} \nu_{12}(\theta)=\lim _{\theta \rightarrow 90^{+}} \nu_{12}(\theta) .
$$

Note that Poisson's ratio $\nu_{12}(\theta)$ has a removable discontinuity point (Thomson et al. 2001) at $\theta=90^{\circ}$, which can be removed by defining Poisson's ratio at $\theta=90^{\circ}$ equal to the limit $L$. This removable discontinuity corresponds to the physical situation in which the structure is locked: for $\theta$ between $0^{\circ}$ and $90^{\circ}$, stretching in the $O x_{1}$ direction will not cause the structure to go past the point when $\theta=90^{\circ}$, as will be the case when $\theta$ is between $90^{\circ}$ and $180^{\circ}$ for compression, which highlights 
the fact that, as discussed elsewhere (Alderson et al. 1997; Smith et al. 1999), the expressions for Poisson's ratios as a function of $\theta$ should not be treated as analogous to relationships of Poisson's ratios with strain.

From equation (2.14), it is important to note that the on-axis Poisson ratios, $\nu_{12}$ and $\nu_{21}$ (for orienting the structure as described earlier) are always negative as $a, b, c, d$ and $\sin (\theta)$ (for $0<\theta<180$ ) are all positive so that the resulting Poisson ratio is negative.

\section{(b) On-axis Young's moduli}

Young's moduli of the structure can be derived through an energy-conservation approach. As we are assuming that the only deformation mechanism is hinging, the stiffness in the structure is solely due to the stiffness of the $\theta$-hinges, which may be described through the stiffness constant $K_{h}$ and defined as

$$
w=\frac{1}{2} K_{h} \mathrm{~d} \theta^{2},
$$

where $w$ is the work done at each hinge in changing the angle $\theta$ by $\mathrm{d} \theta$. As there are four $\theta$-hinges in each unit cell, the total work done is

$$
W=4 w=2 K_{h} \mathrm{~d} \theta^{2} .
$$

The strain energy due to an infinitesimally small strain $\mathrm{d} \varepsilon_{i}$ in the $O x_{i}$ direction is given by

$$
U=\frac{1}{2} E_{i}\left(\mathrm{~d} \varepsilon_{i}\right)^{2},
$$

where $E_{i}$ is Young's modulus of the structure along the $O x_{i}$ direction.

From the principle of conservation of energy,

$$
U=\frac{W}{V}=\frac{2 K_{h} \mathrm{~d} \theta^{2}}{V}
$$

where $V$ is the volume of the unit cell given by

$$
V=X_{11} X_{22} z=[a b+c d+\sin (\theta)(a b+c d)] z,
$$

and $z$ is the out-of-plane thickness of the rectangles.

By equating the two expressions for $U$, Young's moduli are found to be

$$
E_{1}=\frac{4 K_{h} l_{2}^{4} V}{z^{2} \cos ^{2}(\theta)\left[a d^{3}+c b^{3}+b d \sin (\theta)(a d+c b)\right]^{2}}
$$

and

$$
E_{2}=\frac{4 K_{h} l_{2}^{4}}{V_{b}^{2} d^{2} \cos ^{2}(\theta)}
$$




\section{(c) On-axis shear strain}

As already stated, in general, the unit cell shears upon deformation as the internal angle $\alpha_{12}$ is dependent on $\theta$. This will result in a shear strain $\mathrm{d} \gamma$, which can be defined in terms of $\mathrm{d} \theta$ as follows (Grima 2000; Grima et al. 2007b):

$$
\begin{aligned}
\mathrm{d} \gamma & =\frac{1}{X_{11}}\left[\mathrm{~d} X_{12}-\left(\frac{X_{12}}{X_{22}}\right) \mathrm{d} X_{22}\right] \\
& =\frac{1}{X_{11}}\left(\cos \left(\alpha_{12}\right)\left(\frac{\mathrm{d} l_{1}}{\mathrm{~d} \theta}\right)-l_{1} \sin \left(\alpha_{12}\right)\left(\frac{\mathrm{d} \alpha_{12}}{\mathrm{~d} \theta}\right)-\frac{X_{12}}{l_{2}}\left(\frac{\mathrm{d} l_{2}}{\mathrm{~d} \theta}\right)\right) \mathrm{d} \theta
\end{aligned}
$$

where

$$
X_{12}=l_{1} \cos \left(\alpha_{12}\right) \quad \text { and } \quad \cos \left(\alpha_{12}\right)=\frac{\cos (\theta)[a d-c b]}{l_{1} l_{2}} .
$$

Substituting for $X_{11}, X_{12}, \sin \left(\alpha_{12}\right)$ and $\cos \left(\alpha_{12}\right)$ in equation (2.23) and simplifying, the shear strain can be written as

$$
\mathrm{d} \gamma=\frac{z(c b-a d)}{V l_{2}^{2}}\left[2 b d+\left(b^{2}+d^{2}\right) \sin (\theta)\right] \mathrm{d} \theta .
$$

\section{(d) On-axis shear modulus}

The shear modulus $G_{12}$ can be derived using a similar approach used to derive Young's modulus. The shear strain energy due to an infinitesimally small shear strain $\mathrm{d} \gamma$ is related to the total energy $U$ stored in the system through the following equation:

$$
U=\frac{2 K_{h} \mathrm{~d} \theta^{2}}{V}=\frac{1}{2} G_{12}(\mathrm{~d} \gamma)^{2},
$$

such that the shear modulus may be written as

$$
G_{12}=\frac{4 K_{h} l_{2}^{4} V}{z^{2}(a d-c b)^{2}\left[2 b d+\left(b^{2}+d^{2}\right) \sin (\theta)\right]^{2}} .
$$

(e) On-axis shear coupling coefficients

The shear coupling coefficients $\eta_{i j}$ (defined by equation (2.8)) are given by

and

$$
\left.\begin{array}{l}
\eta_{13}=\frac{1}{\eta_{31}}=\frac{(c b-a d)\left[2 b d+\left(b^{2}+d^{2}\right) \sin (\theta)\right]}{\cos (\theta)\left[a d^{3}+c b^{3}+b d \sin (\theta)(a d+c b)\right]} \\
\eta_{23}=\frac{1}{\eta_{32}}=\frac{z(c b-a d)\left[2 b d+\left(b^{2}+d^{2}\right) \sin (\theta)\right]}{V b d \cos (\theta)} .
\end{array}\right\}
$$

\section{(f) Off-axis properties}

The off-axis mechanical properties of the general rotating different-sized rectangles structure may be computed using standard axis-transformation 
techniques (Nye 1957). In particular, Poisson's ratio for loading in any arbitrary direction in the $x-y$ plane (specifically at an angle $+\xi$ to the $x$-axis) is given by

$$
\begin{aligned}
\nu_{12}^{\xi}= & {\left[\frac{\nu_{12} \cos ^{4}(\xi)}{E_{1}}-\cos ^{3}(\xi) \sin (\xi)\left(\frac{\eta_{32}}{G_{12}}-\frac{\eta_{13}}{E_{1}}\right)-\cos ^{2}(\xi) \sin ^{2}(\xi)\left(\frac{1}{E_{1}}+\frac{1}{E_{2}}-\frac{1}{G_{12}}\right)\right.} \\
& \left.-\cos (\xi) \sin ^{3}(\xi)\left(\frac{\eta_{31}}{G_{12}}-\frac{\eta_{23}}{E_{2}}\right)+\frac{\nu_{21} \sin ^{4}(\xi)}{E_{2}}\right] E_{1}^{\xi},
\end{aligned}
$$

where

$$
\begin{aligned}
E_{1}^{\xi}= & {\left[\frac{\cos ^{4}(\xi)}{E_{1}}+\cos ^{3}(\xi) \sin (\xi)\left(\frac{\eta_{31}}{G_{12}}+\frac{\eta_{13}}{E_{1}}\right)-\cos ^{2}(\xi) \sin ^{2}(\xi)\left(\frac{\nu_{12}}{E_{1}}+\frac{\nu_{21}}{E_{2}}-\frac{1}{G_{12}}\right)\right.} \\
& \left.+\cos (\xi) \sin ^{3}(\xi)\left(\frac{\eta_{23}}{E_{2}}+\frac{\eta_{32}}{G_{12}}\right)+\frac{\sin ^{4}(\xi)}{E_{2}}\right]^{-1} .
\end{aligned}
$$

\section{Discussion}

The model presented earlier, which was validated using the Empirical modelling using dummy atoms (EMUDA) methodology, as described by Grima et al. (2005c), suggests that the systems presented here can exhibit a wide range of Poisson's ratios and moduli, which can be fine-tuned to particular pre-desired values through careful choice of the geometric parameters $a, b, c, d$ and $\theta$. This is very significant as it provides us with a key to understand better the requirements for maximizing auxeticity for any particular system.

Let us now present some important observations that apply to the general model presented here (and hence also to all the special cases discussed in appendix A of the electronic supplementary material) by discussing in more detail the significance of equations (2.14) and (2.30) obtained in $\S 2$ for the on-axis and off-axis Poisson ratios of the generalized model. To facilitate the discussion, plots of Poisson's ratios for typical systems are plotted in figure 3 (on-axis Poisson's ratio $\nu_{12}$ versus angle $\theta$ between rectangles) and in figure 4 (off-axis Poisson's ratio $\nu_{12}^{\xi}$ versus direction of loading $\xi$ ). In figure 3 , a selection of representative ratios for $a: b$ and $c: d$ was made. These include the ratios $2: 1,1: 2,3: 4,4: 3$, $2: 3$ and $3: 2$. To fully exploit all possible combinations, the ratio $a: b: c: d$ was considered, and while fixing the ratio for $a: b$, the ratio for $c: d$ was varied. Note that if the ratio of $a: b$ is interchanged with that for $c: d$ (i.e. $a: b: c: d=2: 1: 3: 4$ and $3: 4: 2: 1$ ), this would result in the same structure, i.e. one would obtain the same Poisson ratio plots.

Figure 3 clearly illustrates that Poisson's ratio $\nu_{12}$ is always negative for loading on-axis, irrespective of the size and the degree of openness of the systems. However, the exact value of Poisson's ratio will depend on the geometry of the system. It should also be noted that as $\nu_{21}=\left(\nu_{12}\right)^{-1}, \nu_{21}$ is also always negative for all values of $\theta$.

Moreover, as $\theta$ approaches $90^{\circ}$, the gradient of the curves for $\nu_{12}$ against $\theta$ (and also $\nu_{21}$ against $\theta$ ) approaches zero, resulting in a 'turning point' at the point of the removable discontinuity $\left(\theta=90^{\circ}\right)$, which corresponds to the point at which the rigid units cease to rotate. Referring to figure $5 a$, for any value of 

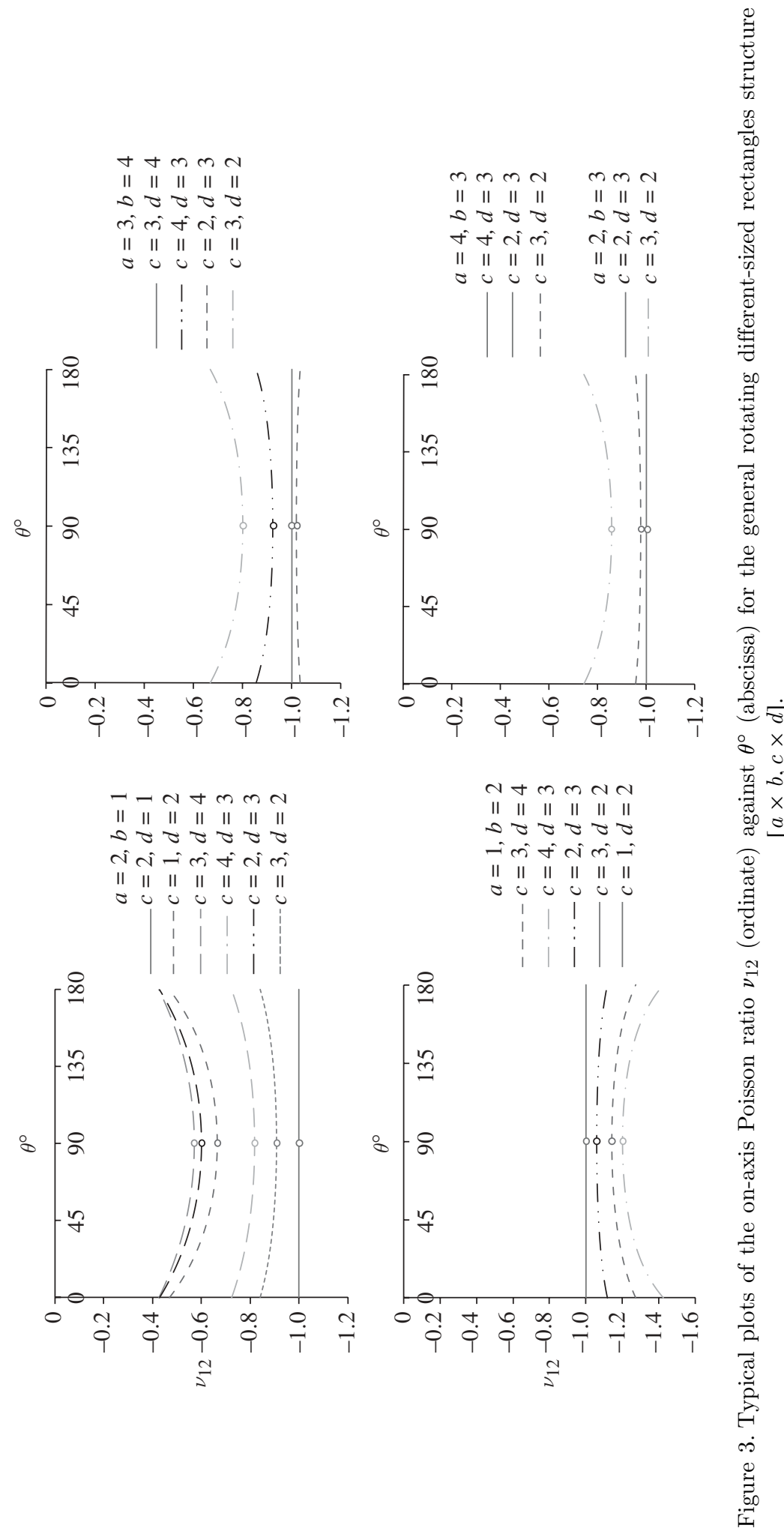

Proc. R. Soc. A (2011) 

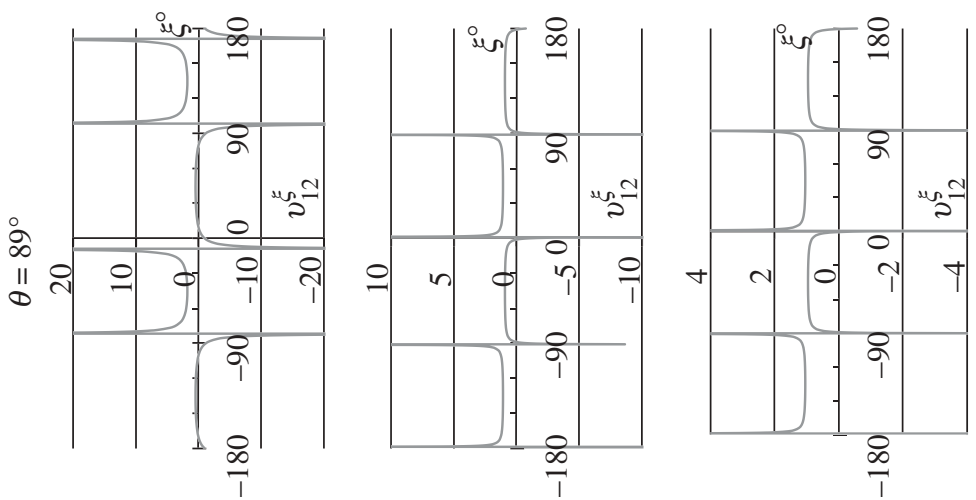

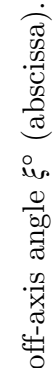
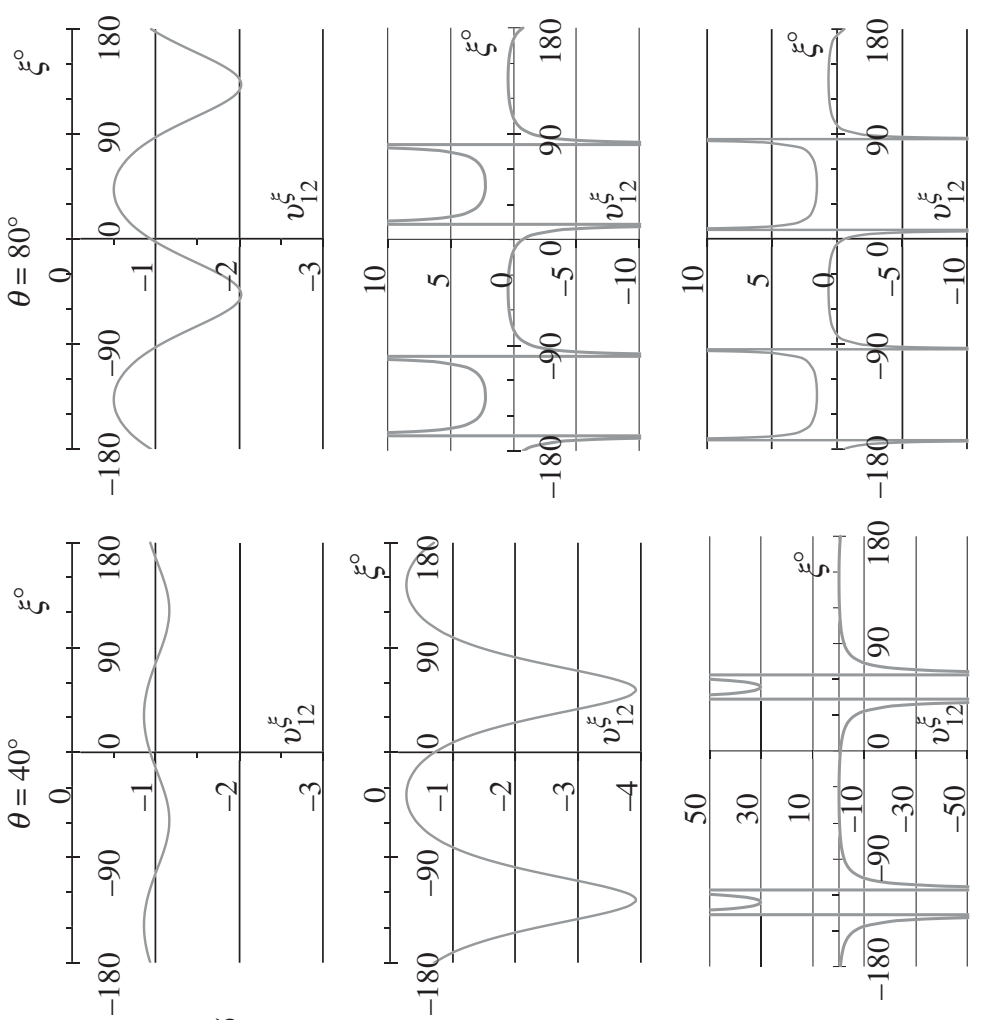

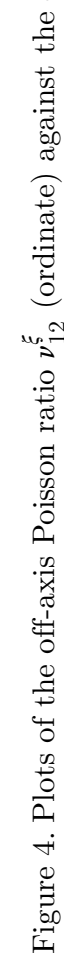

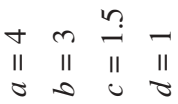

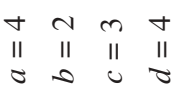

$\begin{array}{cccc}n & 0 & 0 & 0 \\ \| & \| & I & \end{array}$ 
(a)

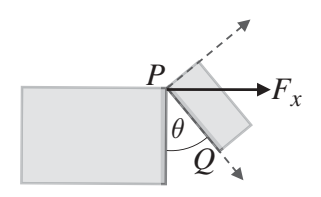

(b)

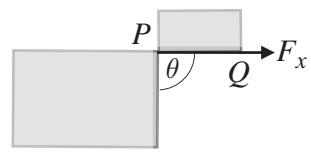

(c)

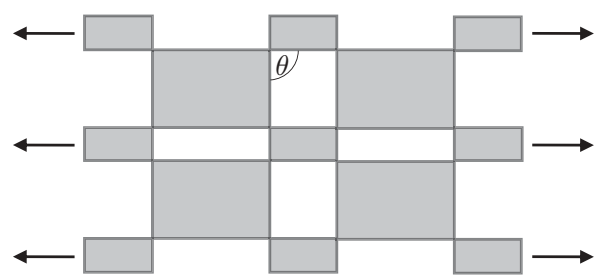

Figure 5. (a) When $\theta<90^{\circ}$, applying a force along the $x$-axis will result in a component of force perpendicular to side $P Q$ creating a moment. (b) When $\theta=90^{\circ}$, there is no component of the force perpendicular to side $P Q$ such that the structure is locked and $(c)$ the locked (fully open) structure.

$\theta$ other than $90^{\circ}$, a stress applied along the $O x_{1}$ direction will result in a force component that is perpendicular to the side $P Q$, resulting in a moment that causes the rectangular units in the structure to rotate. This is not the case when $\theta=90^{\circ}$ (figure $5 b$ ), at which point a stress applied along the $O x_{1}$ direction will result in a force parallel to the edge $P Q$, and hence there is no force component perpendicular to side $P Q$ to create a moment. At this instant, the structure is 'fully open' and becomes locked (figure $5 c$ ), i.e. further tensile loading in the $O x_{1}$ direction will not result in any deformation if one assumes that the rectangles are perfectly rigid (i.e. no other mode of deformation can take place). Thus, loading in the $O x_{1}$ direction will not result in a change in the unit-cell dimensions, a property which in equations $(2.12)$ and $(2.13)$ is represented by the fact that there is a factor $\cos (\theta)$ in the numerator of both expressions of $\mathrm{d} \varepsilon_{i}$, which result in $\mathrm{d} \varepsilon_{i}=0$ when $\theta=90^{\circ}$. The same applies when loading in the $O x_{2}$ direction. Moreover, it should be noted that it is still possible for the system to exist at angles $\theta$ in the range $90^{\circ}<\theta \leq 180^{\circ}$; however, the transition between the regions $0^{\circ} \leq \theta<90^{\circ}$ and $90^{\circ}<\theta \leq 180^{\circ}$ requires forcing the rectangles to rotate past this barrier of $\theta=90^{\circ}$ by, for example, rotating one of the rectangles or in some cases by shearing. Note that at $\theta=180^{\circ}$, the system is 'fully closed', i.e. the sides of the rectangles are touching such that unless overlapping of the rectangles is allowed, $\theta$ cannot be greater than $180^{\circ}$.

Figure 3 also confirms that in the region $0^{\circ} \leq \theta \leq 180^{\circ}$,

- there is just one turning point at $90^{\circ}$ in the relationship $\nu_{i j}=\nu_{i j}(\theta)$ and

- the relationship is symmetric about the line $\theta=90^{\circ}$.

This means that unless $\nu_{i j}=-1$ for all $\theta$ (something that will occur in the special cases discussed subsequently and in appendix $\mathrm{A}$ of the electronic supplementary material), Poisson's ratio will be dependent on the value of $\theta$ with maximum auxeticity either occurring at the points $\theta=0^{\circ}$ and $180^{\circ}$ or at $\theta=90^{\circ}$, depending on the geometry of the system. In fact, it may be shown that the nature of the turning point at $90^{\circ}$ depends on whether $a d^{3}+c b^{3}$ is greater than $b d(a b+c d)$ or smaller. In fact,

- if $a d^{3}+c b^{3}>b d(a b+c d)$, then the turning point for $\nu_{12}$ versus $\theta$ is a minimum turning point, which means that maximum auxeticity (i.e. a minimum point) for loading in the $O x_{1}$ direction occurs as $\theta=90^{\circ}$ and 
- if $a d^{3}+c b^{3}<b d(a b+c d)$, then the turning point for $\nu_{12}$ versus $\theta$ is a maximum turning point, which means that minimum auxeticity is obtained at $\theta=90^{\circ}$ and that maximum auxeticity occurs at the boundaries, i.e. at $\theta=0^{\circ}$ and at $\theta=180^{\circ}$.

It should also be noted that a maximum turning point for $\nu_{12}$ versus $\theta$ corresponds to a minimum turning point for $\nu_{21}$ versus $\theta$ since $\nu_{21}=\left(\nu_{12}\right)^{-1}$. All this suggests that to maximize on-axis auxeticity for loading in the $O x_{1}$ direction, the structure must be such that the sides of the rectangles satisfy $a d^{3}+c b^{3}<b d(a b+c d)$. In such cases, the on-axis Poisson ratio $\nu_{12}$ can assume values that are lower than -1 . To maximize on-axis auxeticity for loading in the $O x_{2}$ direction, the structure must be such that the sides of the rectangles satisfy $a d^{3}+c b^{3}>b d(a b+c d)$. In such cases, the greater the difference between the ratios $a / b$ and $c / d$ where $b \neq d$, the greater the auxeticity.

If one looks at the dependence of Poisson's ratio on the direction of loading, first of all one should note that for any particular structure, the plot showing variation of the off-axis Poisson ratio with $\xi$ at any particular angle $\theta$ is the mirror image of the plot for the same structure when the angle is $180^{\circ}-\theta$. This is to be expected since the systems themselves are also mirror images of each other. Also the off-axis Poisson ratio is obviously periodic with a period of $180^{\circ}$. This is because loading at an angle $+\xi$ is the same as loading at an angle of $\xi-180^{\circ}$.

The plots of the off-axis Poisson ratio $\nu_{12}^{\xi}$ against $\xi$ (figure 4) suggest that when $a / b$ is almost equal to $c / d$, Poisson's ratio $\nu_{12}^{\xi}$ is negative for loading at any off-axis angle, except as $\theta$ approaches $90^{\circ}$, in which case, there are both continuous and asymptotic transitions from negative to positive Poisson ratios and vice versa. Also interesting is the limiting case where $a / b$ is equal to $c / d$ (i.e. similar rectangles structure $[(b c / d) \times b, c \times d])$, for which as discussed below and in appendix A of the electronic supplementary material, Poisson's ratio is equal to -1 and such a structure is isotropic. As the difference between the values of $a / b$ and $c / d$ increases, continuous and asymptotic transitions start to be observed at smaller $\theta$. The greater the difference between the ratios $a / b$ and $c / d$, the smaller the $\theta$, for which both positive Poisson ratios and NPRs are observed. The plots also suggest that for $\theta$ closer to $90^{\circ}$, the range of positive Poisson ratios increases.

Various special cases, which arise from particular combinations of size and aspect ratios of the rectangles in the generalized model, are presented in appendix A of the electronic supplementary material, in which we substitute into and simplify the equations derived in $\S 2$ to consider the special case of systems made from squares of the same size $[a \times a, a \times a]$ (discussed earlier by Grima et al. 1999; Grima 2000; Grima \& Evans 2000a); rectangles of the same size of type I $[a \times b, b \times a]$ and type II $[a \times b, a \times b]$ (discussed earlier by Grima et al. 2004, 2005a,b); systems composed of rotating different-sized squares $[a \times a, c \times c]$ (figure $6 a$ ); systems composed of similar rectangles, where either $a / b=c / d$, i.e. structure $[(b c / d) \times b, c \times d]$ (figure $6 d$ ), or $a / b=d / c$, i.e. structure $[(b d / c) \times b, c \times d]$ (figure $6 c$ ); systems made of rectangles of dimensions $a \times d$ and $c \times d[a \times d, c \times d]$ (figure $6 e$ ) and systems composed of rectangles and squares, i.e. structure $[a \times b, d \times d]$ (figure $6 b$ ). Animations of these cases are available in the electronic supplementary material. 
(a)

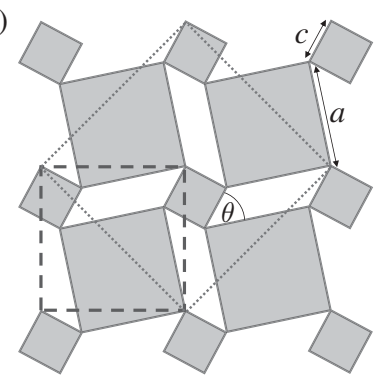

(d)

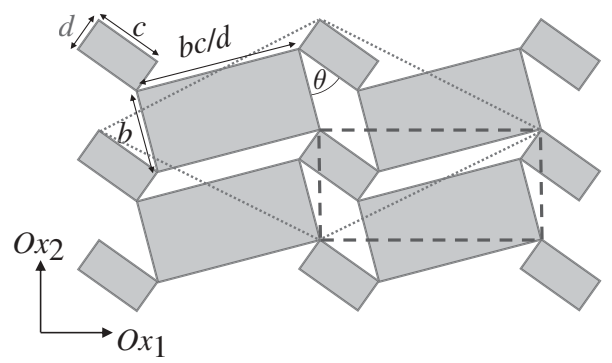

(b)

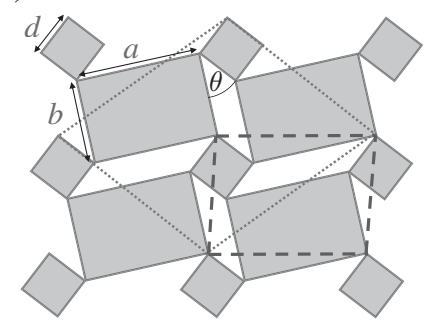

(c)

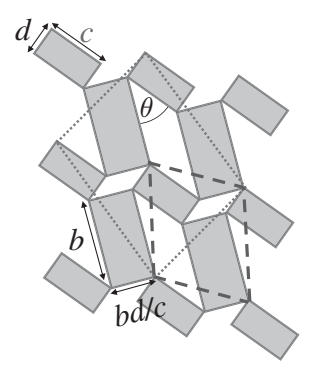

(e)

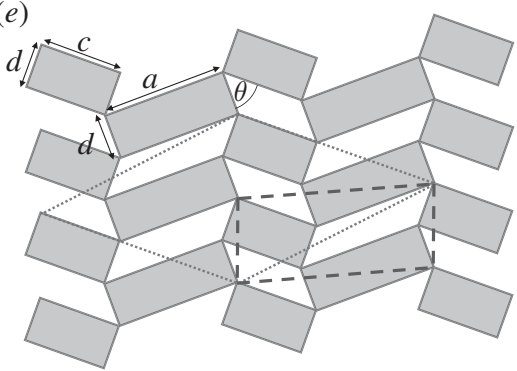

Figure 6. Special cases of the different-sized rotating rectangles structure considered in appendix A of the electronic supplementary material: $(a)$ rotating different-sized squares $[a \times a, c \times c],(b)$ rotating squares and rectangles structure $[a \times b, d \times d],(c)$ similar rectangles $[b d / c \times b, c \times d]$, $(d)$ similar rectangles $[b c / d \times b, c \times d]$ and $(e)$ rotating different-sized rectangles of dimensions $a \times d$ and $c \times d ;[a \times d, c \times d]$ structure. Animations that illustrate how these systems deform when loading in the $O x_{1}$ direction are also supplied (see Anim-6a.gif, Anim-6b.gif, Anim-6c.gif, Anim-6d.gif and Anim-6e.gif, respectively, in the electronic supplementary material). Dotted line, $\mathrm{UC} 1$; dashed line, UC2.

In the case of the same-sized squares structure $[a \times a, a \times a]$ and the type II rectangles [ $a \times b, a \times b]$, Poisson's ratio is -1 for all values of $\theta$ (which means that the Poisson ratio of such a system is strain independent) and for all directions of loading (which suggest that such structures are isotropic in the plane of the structure). This is in accordance with previous work (Grima 2000; Grima \& Evans 2000a; Grima et al. 2005a). For the type I $[a \times b, b \times a]$ rectangles, the derived equations for the on-axis Poisson ratio complement those derived by Grima et al. $(2004,2005 b)$, who derived the properties of this system in a different orientation. The two sets of expressions can be transformed to one another through standard axis-transformation techniques. Here, one should highlight the fact that Grima's earlier derivation had shown that the sign of Poisson's ratio in the directions corresponding to the lattice vector when using UC1 was dependent on the angle between the rectangles, something that is not found for the orientation used here where the on-axis Poisson ratios are always negative. This highlights the necessity to examine the anisotropy when looking for auxeticity.

As noted earlier and as discussed in appendix A of the electronic supplementary material, when the rectangles are similar in such a way that the ratio of the sides $a / b$ is equal to $c / d$, (i.e. [( $b c / d) \times b, c \times d]$ structure), then such a structure is also two-dimensionally isotropic with a Poisson ratio equal to -1 . Special cases of this 
structure are the type II rectangles and the same-sized and different-sized squares (the ratio $a / b$ is equal to 1 in these latter cases). In fact, the mechanical properties of these structures are analogous to that of the 'parent' case, i.e. Poisson's ratio is -1 and these structures are all isotropic.

Another interesting case is the structure $[a \times d, c \times d]$. In this case, the on-axis Poisson ratio is -1 , but such a structure is anisotropic. Note that a special case of this structure is the type II rectangles (structure $[a \times b, a \times b]$ ), which is isotropic.

Let us now discuss some situations in which the model presented here may be used. An important observation that applies to the general model presented here (and hence also to all the special cases discussed in appendix $\mathrm{A}$ of the electronic supplementary material) is that all expressions derived for the mechanical properties are scale independent since if one lets $b=r_{1} a, c=r_{2} a$ and $d=r_{3} a$, then the expression can be re-written in terms of only $r_{i}$ and $\theta$. This means that the deformation mechanism presented here can be implemented at different scales of structures, ranging from the nano (molecular) scale to the macroscale, i.e. the model can be used to predict the behaviour of systems and even design new systems that mimic its geometry and deformation on a smaller scale. On a nanolevel, the usefulness of these models has already been pointed out in other work (Ishibashi \& Iwata 2000; Grima et al. 2005b, 2006, 2007a,c, 2009b; Williams et al. 2007), in which it has been shown that these models can explain, predict, as well as quantify, auxeticity in certain crystalline materials such as $\alpha$-cristobalite (Grima et al. 2006) and natrolite (Grima et al. 2007c, 2009b; Williams et al. 2007) for loading in particular directions. This is possible because the relevant two-dimensional projections of these crystals have the same geometry as the rotating rectangles/squares model, and molecular-modelling simulations also suggest that these molecular systems deform with the same rotating mechanism (Grima et al. 2005b, 2006; Williams et al. 2007).

However, these earlier models based on rotating squares or rectangles were somewhat limited because they required all rectangles/squares to be of the same size. The model presented here has more degrees of freedom, allowing for variations in the size of adjacent units; therefore, one expects that this model may be applied to a wider range of materials. For example, it is interesting to note that at the extremity of having one set of rectangles with a very large aspect ratio relative to the other, then, as shown in figure 7 , the resulting network would bear resemblance to the mechanisms proposed by He et al. $(2005 a, b)$ to explain auxeticity at the nanolevel in liquid-crystalline polymers. At this extremity, the model can also be considered as a network of interconnected nodules and fibrils, which could be of use to model the properties of auxetic microstructured polymers, which have long been studied through nodule-fibril models (Alderson \& Evans 1995, 1997; Alderson et al. 1998b).

Furthermore, the highly versatile model presented here can be of use to researchers who may use it as a 'blueprint' on which they can design and synthesize newly 'designed' auxetic materials. For example, a direct application of this work would be as a template for the design and manufacture of perforated sheets, which exhibit NPR in analogy to the recent work based on squares (Grima \& Gatt 2010). On a microlevel, microstructures based on these models can be micromachined using techniques such as laser ablation and mechanical micromachining. 
(a)

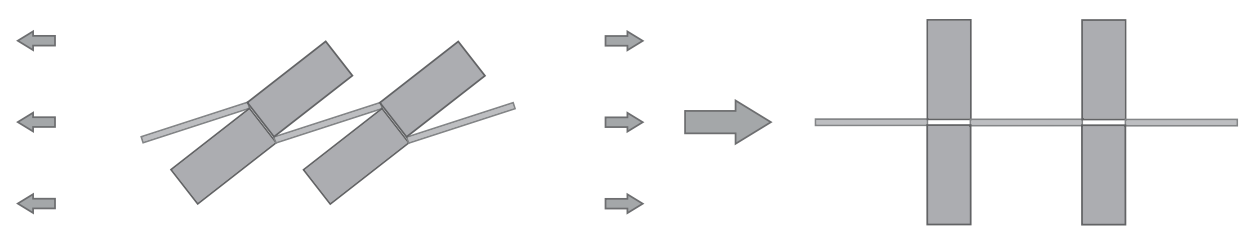

(b)
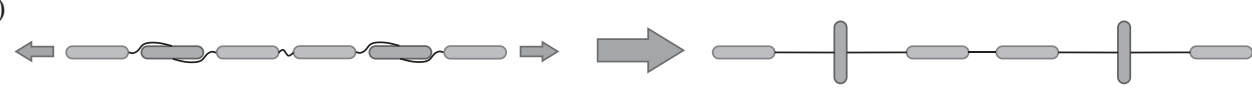

Figure 7. Comparison of the model presented here $(a)$ with that proposed by Griffin et al. (He et al. $2005 a, b)$ to explain auxeticity in liquid-crystalline polymers $(b)$.

Another interesting application of such structures would be the possibility of using them as tunable filters and sieves. The pore size would be controlled by the amount of load applied, such that multi-purpose usage could be achieved. Another advantage of these filters over conventional filters is the fact that these can be easily unclogged by stretching the filter, thus increasing the lifetime of such filters.

Before we conclude, it is important to note some possible limitations of this model (and of other rotating rigid polygons mechanisms in general). Although it has been stated that such networks can be used to predict the mechanical properties of crystalline systems whose two-dimensional projections correspond to the geometry of the systems treated here, one must note that in a real material, it is very unlikely that the projected rectangles are completely rigid, and in addition to rotation, other deformation mechanisms such as stretching may occur concurrently so that the degree of auxeticity would depend on which deformation mechanism dominates. Furthermore, it should be appreciated that at the nanolevel, it is not correct to consider the nanostructure of a material as a purely mechanical system. Thus, at the nanolevel, it is not likely that the extremes of behaviour predicted by this highly idealized model will be manifested to the full. For example, it is not expected that any real nanostructured material will indeed exhibit an infinite shear modulus as predicted by the model of connected rectangles $[a \times b, a \times b]$, although some features of the model would still be present, as, for example, observed in $\alpha$-cristobalite. Besides, one must also keep in mind that the model presented here only offers a two-dimensional representation of three-dimensional systems. In reality, it is the three-dimensional units which are rotating with the result that projected rectangles in the plane of interest may change shape as a result of re-orientation of the three-dimensional units. Such effects are not captured in the model presented here.

\section{Conclusion}

In this work, the on- and off-axis mechanical properties of a structure made up of two different-sized rectangles have been derived. It was shown that some of the systems discussed, in particular, the similar rectangles structure where $a / b$ 
is equal to $c / d$ and the different-sized squares structure, exhibit isotropic and strain-independent Poisson ratios of -1 , whereas some others have Poisson ratios that are dependent on the shape and relative size of the rectangles, the angle between the rectangles and also the direction of loading. It was also shown that since all systems exhibit negative on-axis Poisson ratios, all conformations based on rotating rectangles of this form are auxetic for loading in certain directions. We have also shown that this model could be used to elucidate the behaviour of a wide range of auxetics, ranging from liquid-crystalline polymers to silicates and zeolites.

Given the many benefits associated with having an NPR and the versatility of the proposed systems, it is hoped that this model will stimulate further work. This could, for example, lead to the manufacture of new man-made auxetics, which mimic the behaviour of the model structure proposed here.

\section{References}

Alderson, A. 1999 A triumph of lateral thought. Chem. Ind. 384, 384-391.

Alderson, A. \& Alderson, K. L. 2007 Auxetic materials. Proc. Inst. Mech. Eng., G. J. Aerosp. Eng. 221, 565-575. (doi:10.1243/09544100JAERO185)

Alderson, A. \& Evans, K. E. 1995 Microstructural modeling of auxetic microporous polymers. J. Mater. Sci. 30, 3319-3332. (doi:10.1007/BF00349875)

Alderson, A. \& Evans, K. E. 1997 Modelling concurrent deformation mechanisms in auxetic microporous polymers. J. Mater. Sci. 32, 2797-2809. (doi:10.1023/A:1018660130501)

Alderson, A. \& Evans, K. E. 2001 Rotation and dilation deformation mechanisms for auxetic behaviour in the $\boldsymbol{\alpha}$-cristobalite tetrahedral framework structure. Phys. Chem. Miner. 28, 711-718. (doi:10.1007/s002690100209)

Alderson, A. \& Evans, K. E. 2002 Molecular origin of auxetic behaviour in tetrahedral framework silicates. Phys. Rev. Lett. 89, 225 503-225 507. (doi:10.1103/PhysRevLett.89.225503)

Alderson, K. L., Alderson, A. \& Evans, K. E. 1997 The interpretation of the straindependent Poisson's ratio in auxetic polyethylene. J. Strain Anal. 32, 201-212. (doi:10.1243/ $0309324971513346)$

Alderson, A., Evans, K. E. \& Rasburn, J. 1998 a Smart membrane. International Patent Application no. PCT/GB98/03281, filed November 1998. PCT Patent no. WO 99/22838, published May 1999.

Alderson, K. L., Alderson, A., Webber, R. S. \& Evans, K. E. $1998 b$ Evidence for uniaxial drawing in the fibrillated microstructure of auxetic polymers. J. Mater. Sci. Lett. 17, 1415-1419. (doi:10.1023/A:1026409404057)

Alderson, A., Rasburn, J., Evans, K. E. \& Grima, J. N. 2001 Auxetic polymeric filters display enhanced de-fouling and pressure compensation properties. Membr. Technol. 137, 6-8. (doi:10.1016/S0958-2118(01)80299-8)

Alderson, A., Alderson, K. L., Evans, K. E., Grima, J. N., Williams, M. R. \& Davies, P. J. 2004 Molecular modelling of the deformation mechanisms acting in auxetic silica. Comput. Methods Sci. Technol. 10, 117-126.

Alderson, A., Alderson, K. L., Evans, K. E., Grima, J. N., Williams, M. R. \& Davies, P. J. 2005 Modelling the deformation mechanisms, structure-property relationships and applications of auxetic nanomaterials. Phys. Status Solidi, B Basic Res. 242, 499-508. (doi:10.1002/ pssb.200460370)

Attard, D. \& Grima, J. N. 2008 Auxetic behaviour from rotating rhombi. Phys. Status Solidi, B Basic Res. 245, 2395-2404. (doi:10.1002/pssb.200880269)

Attard, D., Manicaro, E. \& Grima, J. N. 2009a On rotating rigid parallelograms and their potential for exhibiting auxetic behaviour. Phys. Status Solidi, B Basic Res. 246, 2033-2044. (doi:10.1002/ pssb.200982034) 
Attard, D., Manicaro, E., Gatt, R. \& Grima, J. N. $2009 b$ On the properties of auxetic rotating stretching squares. Phys. Status Solidi, B Basic Res. 246, 2045-2054. (doi:10.1002/ pssb.200982035)

Baughman, R. H. \& Galvao, D. S. 1993 Crystalline networks with unusual predicted mechanical and thermal properties. Nature 365, 735-737. (doi:10.1038/365735a0)

Baughman, R. H., Shacklette, J. M., Zakhidov, A. A. \& Stafstrom, S. 1998 Negative Poisson's ratios as a common feature of cubic metals. Nature 392, 362-365. (doi:10.1038/32842)

Bezazi, A. \& Scarpa, F. 2006 Mechanical behaviour of conventional and negative Poisson's ratio thermoplastic polyurethane foams under compressive cyclic loading. Int. J. Fatig. 29, 922-930. (doi:10.1016/j.ijfatigue.2006.07.015)

Caddock, B. D. \& Evans, K. E. 1989 Microporous materials with negative Poisson's ratio I: microstructure and mechanical properties. J. Phys. D 22, 1877-1882. (doi:10.1088/0022-3727/ $22 / 12 / 012)$

Daniel, I. M. \& Ishai, O. 1994 Engineering mechanics of composite materials. New York, NY: Oxford University Press.

Evans, K. E. \& Alderson, A. 2000 Auxetic materials: functional materials and structures from lateral thinking. Adv. Mater. 12, 617-628. (doi:10.1002/(SICI)1521-4095(200005)12:9\% 3C617::AID-ADMA617\%3E3.0.CO;2-3)

Evans, K. E., Nkansah, M. A., Hutchinson, I. \& Rodgers, S. C. 1991 Molecular network design. Nature 353, 124-125 (doi:10.1038/353124a0)

Evans, K. E., Nkansah, M. A. \& Hutchinson, I. J. 1994 Auxetic foams: modelling negative Poisson's ratios. Acta Metall. Mater. 42, 1289-1294. (doi:10.1016/0956-7151(94)90145-7)

Evans, K. E., Alderson, A. \& Christian, F. R. 1995 Auxetic two-dimensional polymer networks: an example of tailoring geometry for specific mechanical properties. J. Chem. Soc. Faraday Trans. 91, 2671-2680. (doi:10.1039/ft9959102671)

Gaspar, N. 2008 Estimates for the localised stiffness and heterogeneity of auxetic foams. Phys. Status Solidi, B Basic Res. 245, 497-501. (doi:10.1002/pssb.200777702)

Gaspar, N., Smith, C. W. \& Evans, K. E. 2003 The effect of heterogeneity on the elastic properties of auxetic materials. J. Appl. Phys. 94, 6143-6149. (doi:10.1063/1.1614847)

Gibson, L. J. \& Ashby, M. F. 1997 Cellular solids: structure and properties, 2nd edn. Cambridge, UK: Cambridge University Press.

Gibson, L. J., Ashby, M. F., Schajer, G. S. \& Robertson, C. I. 1982 The mechanics of twodimensional cellular materials. Proc. R. Soc. Lond. A 382, 25-42. (doi:10.1098/rspa.1982.0087)

Grima, J. N. 2000 New auxetic materials. PhD thesis, University of Exeter, UK.

Grima, J. N. \& Evans, K. E. 2000 a Auxetic behaviour from rotating squares. J. Mater. Sci. Lett. 19, 1563-1565. (doi:10.1023/A:1006781224002)

Grima, J. N. \& Evans, K. E. $2000 b$ Self expanding molecular networks. Chem. Commun. 16, 1531-1532. (doi:10.1039/b004305m)

Grima, J. N. \& Evans, K. E. 2006 Auxetic behaviour from rotating triangles. J. Mater. Sci. 41, 3193-3196. (doi:10.1007/s10853-006-6339-8)

Grima, J. N. \& Gatt, R. 2010 Perforated sheets exhibiting negative Poisson's ratios. Adv. Eng. Mater. 12, 460-464. (doi:10.1002/adem.201000005)

Grima, J. N., Alderson, A. \& Evans, K. E. 1999 Zeolites with negative Poisson's ratios. In RSC 4th Int. Materials Conf. (MC4), Dublin, Ireland, P81, July 1999.

Grima, J. N., Jackson, R., Alderson, A. \& Evans, K. E. 2000 Do zeolites have negative Poisson's ratios? Adv. Mater. 12, 1912-1918. (doi:10.1002/1521-4095(200012)12:24\% 3C1912::AID-ADMA1912\%3E3.0.CO;2-7)

Grima, J. N., Alderson, A. \& Evans, K. E. 2004 Negative Poisson's ratio from rotating rectangles. Comput. Methods Sci. Technol. 10, 137-145.

Grima, J. N., Gatt, R., Alderson, A. \& Evans, K. E. $2005 a$ On the auxetic properties of 'rotating rectangles' with different connectivity. J. Phys. Soc. Jpn 74, 2866-2867. (doi:10.1143/ JPSJ.74.2866)

Grima, J. N., Alderson, A. \& Evans, K. E. $2005 b$ Auxetic behaviour from rotating rigid units. Phys. Status Solidi, B Basic Res. 242, 561-575. (doi:10.1002/pssb.200460376) 
Grima, J. N., Gatt, R., Chircop Bray, T. G., Alderson A. \& Evans, K. E. 2005 c Empirical modelling using dummy atoms (EMUDA): an alternative approach for studying 'auxetic' structures. Mol. Simul. 31, 915-924. (doi:10.1080/08927020500401121)

Grima, J. N., Gatt, R., Alderson, A. \& Evans, K. E. 2006 An alternative explanation for the negative Poisson's ratios in $\alpha$-cristobalite. Mater. Sci. Eng. A 423, 219-224. (doi:10.1016/ j.msea.2005.08.230)

Grima, J. N., Zammit, V., Gatt, R., Alderson, A. \& Evans, K. E. 2007 a Auxetic behaviour from rotating semi-rigid units. Phys. Status Solidi, B Basic Res. 224, 866-882. (doi:10.1002/ pssb.200572706)

Grima, J. N., Farrugia, P. S., Gatt, R. \& Zammit, V. $2007 b$ A system with adjustable positive or negative thermal expansion. Proc. R. Soc. A 463, 1585-1596. (doi:10.1098/rspa. 2007.1841)

Grima, J. N., Gatt, R., Zammit, V., Williams, J. J., Evans, K. E., Alderson, A. \& Walton, R. I. 2007 c Natrolite: a zeolite with negative Poisson's ratios. J. Appl. Phys. 101, 86 102-86105. (doi:10.1063/1.2718879)

Grima, J. N., Farrugia, P. S., Gatt, R. \& Attard, D. 2008a On the auxetic properties of rotating rhombi and parallelograms: a preliminary investigation. Phys. Status Solidi, B Basic Res. 245, 521-529. (doi:10.1002/pssb.200777705)

Grima, J. N., Gatt, R. \& Farrugia, P. S. $2008 b$ On the properties of auxetic meta-tetrachiral structures. Phys. Status Solidi, B Basic Res. 245, 511-520. (doi:10.1002/pssb.200777704)

Grima, J. N., Attard, D., Gatt, R. \& Cassar, R. N. 2009 a A novel process for the manufacture of auxetic foams and for their reconversion to conventional form. Adv. Eng. Mater. 11, 533-535. (doi:10.1002/adem.200800388)

Grima, J. N., Cassar, R. N. \& Gatt, R. $2009 b$ On the effect of hydrostatic pressure on the auxetic character of NAT-type silicates. J. Non-Cryst. Sol. 355, 1307-1312. (doi:10.1016/ j.jnoncrysol.2009.05.018)

He, C., Liu, P., Griffin, A. C., Smith, C. W. \& Evans, K. E. 2005 a Morphology of a liquid crystalline polymer containing laterally attached pentaphenyl rods. Macromol. Chem. Phys. 206, 233-239. (doi:10.1002/macp.200400302)

He, C., Liu, P., McMullan, P. J. \& Griffin, A. C. $2005 b$ Toward molecular auxetics: main chain liquid crystalline polymers consisting of laterally attached para-quaterphenyls. Phys. Status Solidi, B Basic Res. 242, 576-584. (doi:10.1002/pssb.200460393)

Horrigan, E. J., Smith, C. W., Scarpa, F., Gaspar, N., Javadi, A. A., Berger, M. A. \& Evans, K. E. 2009 Simulated optimisation of disordered structures with negative Poisson's ratios. Mech. Mat. 41, 919-927. (doi:10.1016/j.mechmat.2009.04.008)

Ishibashi, Y. \& Iwata, M. 2000 A microscopic model of a negative Poisson's ratio in some crystals. J. Phys. Soc. Jpn 69, 2702-2703. (doi:10.1143/JPSJ.69.2702)

Keskar, N. R. \& Chelikowsky, J. R. 1992 Negative Poisson ratios in crystalline $\mathrm{SiO}_{2}$ from 1stprinciples calculations. Nature 358, 222-224. (doi:10.1038/358222a0)

Kimizuka, H., Kaburaki, H. \& Kogure, Y. 2000 Mechanism for negative Poisson ratios over the alpha-beta transition of cristobalite, $\mathrm{SiO}_{2}$ : a molecular-dynamics study. Phys. Rev. Lett. 84, 5548-5551. (doi:10.1103/PhysRevLett.84.5548)

Lakes, R. S. 1987 Foam structures with a negative Poisson's ratio. Science 235, 1038-1040. (doi:10.1126/science.235.4792.1038)

Lakes, R. S \& Elms, K. E. 1993 Indentability of conventional and negative Poisson's ratio foams. J. Compos. Mater. 27, 1193-1202. (doi:10.1177/002199839302701203)

Masters, I. G. \& Evans, K. E. 1996 Models for the elastic deformation of honeycombs. Compos. Struct. 35, 403-422. (doi:10.1016/S0263-8223(96)00054-2)

Nye, J. F. 1957 Physical properties of crystals, pp. 33-49. New York, NY: Oxford University Press.

Prall, D. \& Lakes, R. S. 1997 Properties of a chiral honeycomb with a Poisson's ratio of -1 . Int. J. Mech. Sci. 39, 305-314. (doi:10.1016/S0020-7403(96)00025-2)

Ravirala, N., Alderson, A., Alderson, K. L. \& Davies, P. J. 2005 Expanding the range of auxetic polymeric products using a novel melt-spinning route. Phys. Status Solidi, B Basic Res. 242, 653-664. (doi:10.1002/pssb.200460384) 
Sanchez-Valle, C., Sinogeikin, S. V., Lethbridge, Z. A. D., Walton, R. I., Smith, C. W., Evans, K. E. \& Bass, J. D. 2005 Brillouin scattering study on the single-crystal elastic properties of natrolite and analcime zeolites. J. Appl. Phys. 98, 053508. (doi:10.1063/1.2014932)

Scarpa, F. \& Smith, F. C. 2004 Passive and MR fluid-coated auxetic PU foam - mechanical, acoustic, and electromagnetic properties. J. Intell. Mater. Syst. Struct. 15, 973-979. (doi:10.1177/1045389X04046610)

Scarpa, F. \& Tomlinson, G. 2000 Theoretical characteristics of the vibration of sandwich plates with in-plane negative Poisson's ratio values. J. Sound Vib. 230, 45-67. (doi:10.1006/jsvi.1999.2600)

Scarpa, F., Ciffo, L. G. \& Yates, Y. R. $2004 a$ Dynamic properties of high structural integrity auxetic open cell foam. Smart Mater. Struct. 13, 49-56. (doi:10.1088/0964-1726/13/1/006)

Scarpa, F., Bullough, W. A. \& Lumley, P. $2004 b$ Trends in acoustic properties of iron particle seeded auxetic polyurethane foam. Proc. Inst. Mech. Eng. C J. Mech. Eng., Sci. 218, 241-244. (doi:10.1243/095440604322887099)

Scarpa, F., Giacomin, J., Zhang, Y. \& Pastorino, P. 2005 Mechanical performance of auxetic polyurethane foam for antivibration glove applications. Cell. Polym. 24, 253-268.

Smith, C. W., Wootton, R. J. \& Evans, K. E. 1999 Interpretation of the experimental data for Poisson's ratio of highly nonlinear materials. Exp. Mech. 39, 356-362. (doi:10.1007/BF02329817)

Spadoni, A., Ruzzene, M. \& Scarpa, F. 2005 Global and local linear buckling behaviour of a chiral cellular structure. Phys. Status Solidi, B Basic Res. 242, 695-709. (doi:10.1002/pssb.200460387)

Thomson, B. S., Bruckner, J. B. \& Bruckner, A. M. 2001 Elementary real analysis. Upper Saddle River, NJ: Prentice-Hall (Pearson).

Williams, J. J., Smith, C. W., Evans, K. E., Lethbridge, Z. D. A. \& Walton, R. I. 2007 An analytical model for producing negative Poisson's ratios and its application in explaining offaxis elastic properties of the NAT-type zeolites. Acta Mater. 55, 5697-5706. (doi:10.1016/ j.actamat.2007.06.011)

Yeganeh-Haeri, A., Weidner, D. J. \& Parise, J. B. 1992 Elasticity of $\alpha$-cristobalite: a silicon dioxide with a negative Poisson's ratio. Science 257, 650-652. (doi:10.1126/science.257.5070.650) 